\title{
SIRT1 keeps escapees quiet
}

Although self-reactive T cells can escape deletion in the thymus, they are controlled in the periphery by mechanisms that induce tolerance, such as clonal anergy, by which $\mathrm{T}$ cells become unresponsive following suboptimal stimulation. The heterodimeric transcription factor activator protein 1 (AP1) is required for $\mathrm{T}$ cell activation and is selectively inhibited during peripheral $\mathrm{T}$ cell tolerance, but the factors governing this inhibition are not known. Zhang et al. now show that NAD-dependent deacetylase sirtuin 1 (SIRT1), a type III histone deacetylase, maintains anergy in T cells by inhibiting acetylation of JUN, a component of AP1.

Previous studies showed that a pharmacological SIRT1 activator suppressed proliferation of and cytokine production by $\mathrm{T}$ cells, which suggested that SIRT1 might be a negative regulator of $\mathrm{T}$ cell activation. To test this, the authors used Sirt1 $1^{-/-}$mice. Although T cell development was normal in these mice, stimulation of Sirt1 $1^{-1-} \mathrm{T}$ cells in vitro and in vivo led to greater proliferation and more interleukin-2 production than stimulation of T cells from Sirt $1^{+/-}$littermates. Interestingly, in vitro stimulation of Sirt $1^{-1-} \mathrm{T}$ cells with CD3-specific antibodies without co-stimulation, which induces anergy in wild-type T cells, resulted in full $\mathrm{T}$ cell activation, suggesting that SIRT1 might function as an anergic factor. A four- to fivefold increase in Sirt1 expression levels in anergic wild-type T cells compared with naive wild-type $\mathrm{T}$ cells provided further evidence of a role for SIRT1 in anergy.

To determine whether SIRT1 was required to maintain $\mathrm{T}$ cell peripheral tolerance to self antigen in vivo, Sirt1 $1^{-/-}$and Sirt1 ${ }^{+/-}$mice

were immunized with myelin oligodendrocyte glycoprotein 35-55 peptide to induce experimental autoimmune encephalomyelitis (EAE). More Sirt $1^{-/-}$mice showed signs of EAE after immunization, disease onset occurred earlier and the average clinical score was higher than for the Sirt1 $1^{+/-}$ mice. So, the authors concluded that SIRT1 prevented activation of autoreactive $\mathrm{T}$ cells and the development of autoimmunity.

Because SIRT1 had previously been shown to suppress several transcription factors, the authors looked at whether SIRT1 could suppress AP1 activity in T cells. They showed that overexpression of SIRT1 inhibited AP1 transcriptional activity in a dose-dependent manner. As SIRT1 is a deacetylase and JUN requires acetylation for its activity it was suggested that SIRT1 might inhibit AP1 by suppressing JUN acetylation. Indeed, there was more acetylation in Sirt $1^{-/-} \mathrm{T}$ cells than in Sirt $1^{+-} \mathrm{T}$ cells and overexpression of SIRT1 inhibited JUN acetylation in transfected HEK-293 cells.

Thus, the findings that SIRT1 is an inhibitor of $\mathrm{T}$ cell activation and is required for tolerance suggest that SIRT1 activators could have therapeutic potential for the treatment of $\mathrm{T}$ cell-mediated autoimmune diseases and other T cell-mediated inflammatory conditions.

Gemma Ryan, Nature Reviews Immunology

ORIGINAL RESEARCH PAPER Zhang, J. et al. The type III histone deacetylase SIRT1 is essential for maintenance of T cell tolerance in mice. J. Clin. Invest. 1 Sep 2009 (doi:10.1172/JCl38902) 